\title{
High-Dose Radiation as a Dramatic, Immunological Primer in Locally Advanced Melanoma
}

\author{
Majid Mohiuddin ${ }^{1}$, Harold Park ${ }^{1}$, Sigrun Hallmeyer ${ }^{2}$, Jon Richards ${ }^{2}$ \\ 1. Radiation Oncology, Advocate Lutheran General Hospital 2. Medical Oncology, Advocate Lutheran \\ General Hospital
}

$\square$ Corresponding author: Majid Mohiuddin, majid.mohiuddin.md@gmail.com Disclosures can be found in Additional Information at the end of the article

\section{Abstract}

A 53-year-old white male presented with a right axillary melanoma that became widely metastatic and progressive despite multiple systemic treatments. He became refractory to ipilimumab (Yervoy) and pembrolizumab (Keytruda). He presented with a very large, painful left posterior neck mass that was $18 \times 15 \times 8 \mathrm{~cm}$ in size clinically. He was treated with a single fraction of 20 Gy using parallel opposed, spatially fractionated GRID radiation therapy (SFGRT), along with concurrent pembrolizumab. He also received $50 \mathrm{~Gy}$ in 25 fractions of conventional radiation. After five months of concurrent treatment, the refractory neck mass had completely resolved and he had no lasting side effects. Our dramatic case confirms the synergistic effect of high-dose GRID radiation as a primer for renewed, enhanced immunological response, and we have used this approach successfully on a number of similar patients with rapid and durable results.

Categories: Radiation Oncology, Allergy/Immunology, Oncology

Keywords: immunotherapy, spatially fractionated radiation therapy (grid), metastatic melanoma, ipilimumab, pembrolizumab

\section{Introduction}

The current treatment management for advanced melanoma attempts to enhance the patient's own immune system to attack evasive cancer cells. Previously, this was done with cytokines, like interleukin-2, to give a general boost at significant toxicity, but recently, considerable progress has been made with the approval of novel immune checkpoint inhibitors in the form of specific human monoclonal antibodies. These newer drugs, such as ipilimumab and

Received 11/23/2015

Review began 11/26/2015

Review ended 12/14/2015

Published 12/18/2015

@ Copyright 2015

Mohiuddin et al. This is an open access article distributed under the terms of the Creative Commons Attribution License CC-BY 3.0., which permits unrestricted use, distribution, and reproduction in any medium, provided the original author and source are credited. pembrolizumab, target and inhibit proteins on T-cells, such as CTLA-4 or PD-1, respectively. By removing a suppressive checkpoint on the immune response, the immune response is enhanced against the cancer cells. Pembrolizumab has shown increased progression-free survival as compared to chemotherapy in ipilimumab-refractory patients, but unfortunately, the response is only seen in a subset of patients [1]. When patients are ineligible for these treatments due to the tumor's genetic signature, or later prove refractory or unresponsive to these kinds of immunotherapies, they have few options left. We report on a pembrolizumab-refractory patient with an enlarging disease who was dramatically re-sensitized to the same drug by the administration of high-dose radiation.

\section{Case Presentation}

Informed patient consent was obtained. No identifying patient information was disclosed in 


\section{Cureus}

this paper.

A 53-year-old white male originally presented with a Stage IIIA right shoulder and right axillary melanoma and underwent wide local excision and lymph node dissection in August 2011. Two years later, he developed a painful retroperitoneal disease. In April 2013, he underwent surgical resection of the spleen, right adrenal gland, bowel, and kidney that proved he had metastatic melanoma, BRAF-negative.

In June 2013, he started ipilimumab (Yervoy) due to a new, large posterior left neck mass and disease in the retroperitoneum. By August 2013, the ipilimumab was discontinued after four cycles due to dermatitis requiring steroids. The neck mass and retroperitoneal disease responded partially at first, but then grew back by October 2013.

By January 2014, the neck mass demonstrated 15\% further progression. Therefore, the patient was started on high-dose interleukin-2 (IL-2) for two cycles, but he still had progression. He was started on the anti-PD-1 directed monoclonal antibody, pembrolizumab (Keytruda), for five cycles from June to September 2014 but still showed a significant progression of an additional 30\% by RECIST (response evaluation criteria in solid tumors) criteria.

In September 2014, he presented with a left posterior neck mass that was $18 \mathrm{~cm}$ in the anteriorposterior dimension, $15 \mathrm{~cm}$ superior-inferior, and $8 \mathrm{~cm}$ thick causing pain and a decreased range of motion (Figure 1).
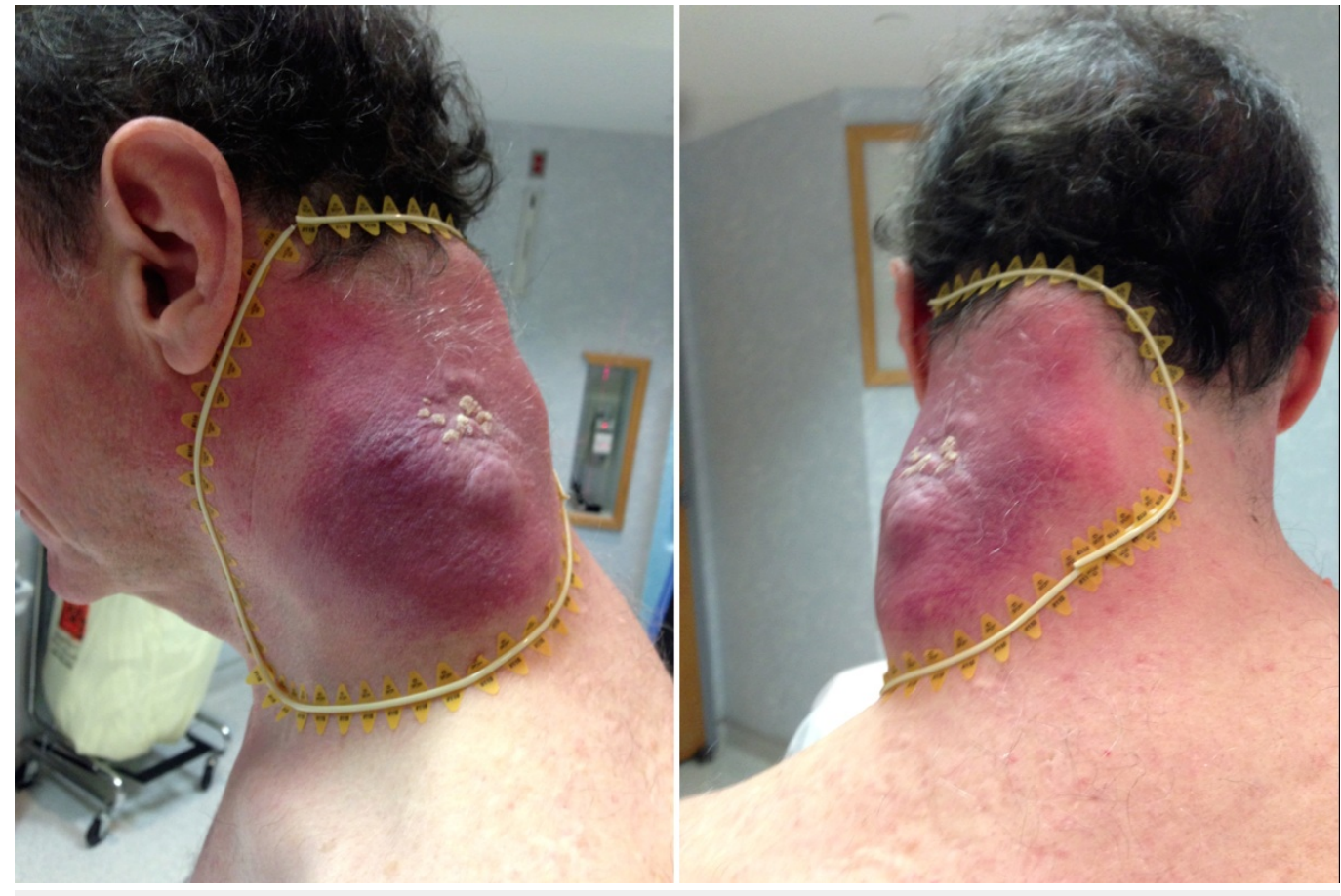

\section{FIGURE 1: Initial Tumor on Neck}

The initial tumor on the left posterior neck of $18 \times 15 \times 8 \mathrm{~cm}$ size, demarcated by wire.

The tumor volume was $650 \mathrm{~cm}^{3}$ (10.7 cm equivalent sphere diameter) prior to treatment. He received $20 \mathrm{~Gy}$ in a single fraction using parallel opposed spatially fractionated GRID radiation therapy (SFGRT) to the left neck through a commercially available, specialized brass block (dot 


\section{Cureus}

Decimal Inc., Sanford, FL). The GRID block treated the large tumor with high-dose beamlets spatially arranged in a 50:50 open-to-closed ratio (Figure 2).

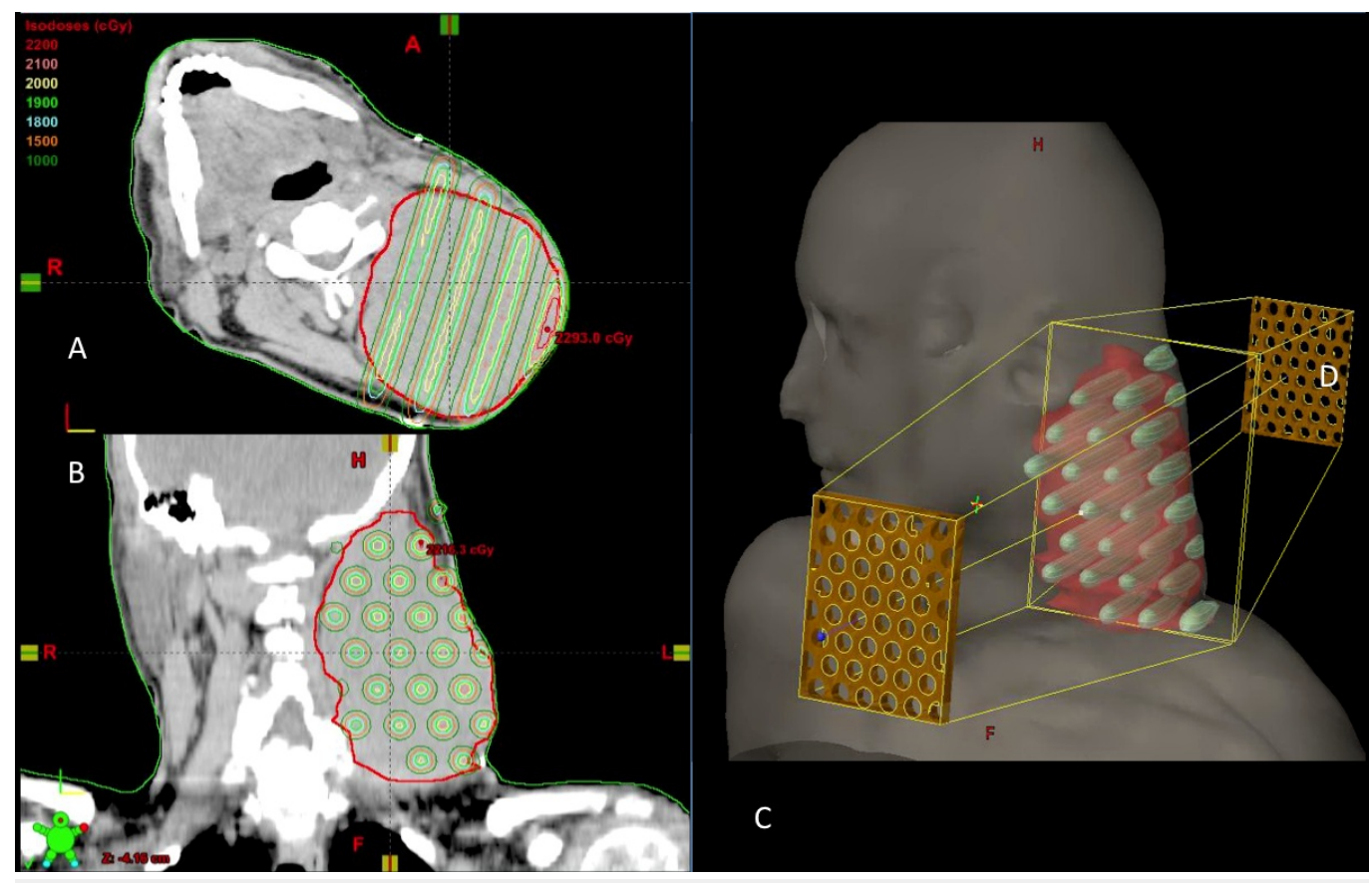

\section{FIGURE 2: Radiation Dosimetry with GRID}

The initial tumor (red line) treated by GRID radiation of 20 Gy (yellow lines) in the A) axial, B) coronal, and C) 3-dimensional view with GRID blocks shown.

This was followed by $50 \mathrm{~Gy}$ in 25 fractions of conventional radiation to the left neck completed by October 2014. The radiation was given with concurrent pembrolizumab. He had no side effects from the combination treatment.

Two months after the treatment to the neck, the tumor mass had regressed over $75 \%$ by tumor volume, and clinically, he had a full range of neck motion and no adverse side -effects. By five months, there was complete resolution of the neck mass on CT scan (Figure 3).

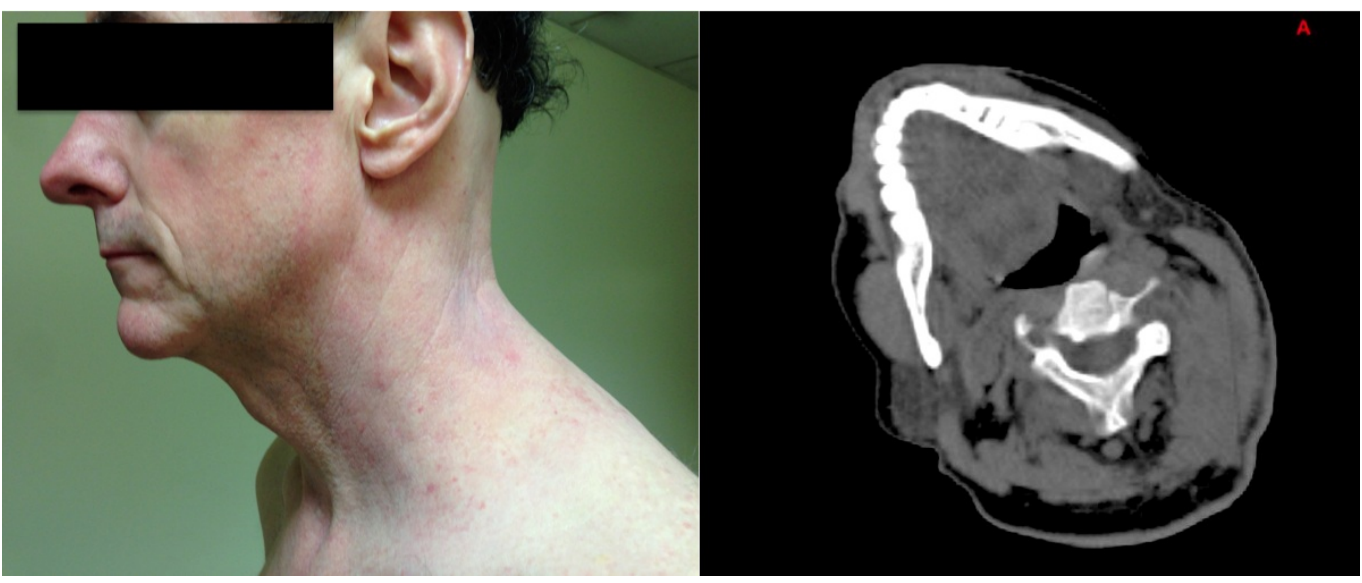

FIGURE 3: Tumor Response to Treatment 
The pembrolizumab was continued for a total of 18 cycles from June 2014 to June 2015. The retroperitoneal disease remained stable for six months and then started to progress. There has been no recurrence of tumor in the neck over the last 12 months from radiation.

\section{Discussion}

We present a locally advanced melanoma tumor that initially responded to ipilimumab, but then progressed and became refractory to multiple systemic, immunological agents, including ipilimumab, high-dose IL-2, and pembrolizumab. We then introduced high-dose radiation along with the anti-PD-1 agent, pembrolizumab, and the patient had a rapid, complete response to treatment in the neck with minimal side effects.

The patient's tumor had previously progressed on five cycles of pembrolizumab alone prior to radiation. Conventional radiation of 50 Gy alone would not generate the dramatic, rapid response seen here, either. Our case is important because it suggests that high-dose radiation can be used as an immunological primer for re-introducing sensitivity to biological agents. Formenti, et al. has suggested that the local radiation on the patient's tissue acts as an in-situ vaccine [2]. Radiation targeted to the intact primary tumor can release radiation-specific antigens and induce attracting chemokines to activated T-cells, thus engaging the patient's innate immune response against the tumor [3-4]. If the tumor-specific immune response is strong enough, it can also enable systemic regression in areas outside of the local radiation field, known as the abscopal effect [4].

Spatially fractionated GRID radiation therapy (SFGRT) is an ideal method for delivering a high dose of priming radiation to large-sized tumors with minimal side effects. It has been used with clinical success in a variety of radio-resistant tumors like melanoma and sarcoma [5]. The GRID block is made of $1.43 \mathrm{~cm}$ diameter circles spaced $2.11 \mathrm{~cm}$ apart (center-to-center) at 60-degree angles that allow delivery of multiple, very high-dose radiation beamlets to the tumor while also sparing considerable skin in the blocked areas. In a sense, it is similar to stereotactic body radiation therapy (SBRT), given its high dose per fraction, and it is like "non-invasive" interstitial brachytherapy, given its spatial dose delivery over a large area.

In short, the synergism of immunotherapy and local radiation dramatically upregulates the host's immune response, even in tumors that were previously refractory to immune therapy. We have treated other locally advanced and refractory patients using this paradigm with similar success. Randomized trials are ongoing combining radiation with checkpoint blockade.

\section{Conclusions}

Spatially fractionated GRID radiation therapy (SFGRT) delivers a high dose of radiation to large-sized tumors with minimal side effects. When combined with immunotherapy like the anti-PD-1 monoclonal antibody, pembrolizumab (Keytruda), it can prime the patient's refractory tumor to provide a new and dramatic synergistic immune response. Randomized trials are ongoing combining radiation with checkpoint blockade.

\section{Additional Information}

\section{Disclosures}

Human subjects: Consent was obtained by all participants in this study. Conflicts of interest: 
In compliance with the ICMJE uniform disclosure form, all authors declare the following:

Payment/services info: All authors have declared that no financial support was received from any organization for the submitted work. Financial relationships: All authors have declared that they have no financial relationships at present or within the previous three years with any organizations that might have an interest in the submitted work. Other relationships: All authors have declared that there are no other relationships or activities that could appear to have influenced the submitted work.

\section{References}

1. Ribas A, Puzanov I, Dummer R, Schadendorf D, Hamid O, Robert C, Hodi FS, Schachter J, Pavlick AC, Lewis KD, Cranmer LD, Blank CU,O'Day SJ, Ascierto PA, Salama AK, Margolin KA, Loquai C, Eigentler TK, Gangadhar TC, Carlino MS, Agarwala SS, Moschos SJ, Sosman JA, Goldinger SM, Shapira-Frommer R, Gonzalez R, Kirkwood JM, Wolchok JD, Eggermont A, Li XN, Zhou W, Zernhelt AM, Lis J,Ebbinghaus S, Kang SP, Daud A: Pembrolizumab versus investigator-choice chemotherapy for ipilimumab-refractory melanoma (KEYNOTE-002): a randomised, controlled, phase 2 trial. Lancet Oncol. 2015, 16:908-18. 10.1016/S14702045(15)00083-2

2. Formenti SC, Demaria S: Radiation therapy to convert the tumor into an in situ vaccine . Int J Radiat Oncol Biol Phys. 2012, 84:879-80. 10.1016/j.ijrobp.2012.06.020

3. Reits EA, Hodge JW, Herberts CA, Groothuis TA, Chakraborty M, Wansley EK, Camphausen K, Luiten RM, de Ru AH, Neijssen J, Griekspoor A, Mesman E, Verreck FA, Spits H, Schlom J, van Veelen P, Neefjes JJ: Radiation modulates the peptide repertoire, enhances MHC class I expression, and induces successful antitumor immunotherapy. J Exp Med. 2006, 203:1259-71. 10.1084/jem.20052494

4. Formenti SC, Demaria S: Systemic effects of local radiotherapy . Lancet Oncol. 2009, 10:71826. 10.1016/S1470-2045(09)70082-8

5. Kaiser A, Mohiuddin MM, Jackson G: Dramatic response from neoadjuvant, spatially fractionated GRID radiotherapy (SFGRT) for large, high-grade extremity sarcoma. J Radiat Oncol. 2013, 2:103-106. 10.1007/s13566-012-0064-5 